\title{
Top quark and electroweak results from CDF
}

\author{
Sandra Leone ${ }^{\mathrm{a}}$ \\ (for the CDF Collaboration)

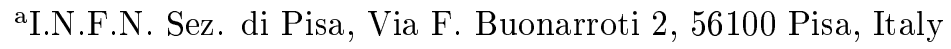

In 2001 the Tevatron run II began, after a five year period of significant upgrade of the accelerator itself and of the experiments CDF and DØ. After a detector commissioning run, the CDF experiment is now taking high quality data with all subsystems functional. We report in this talk the first preliminary CDF results on top quark and $\mathrm{W} / \mathrm{Z}$ boson properties, based on run II data. The top quark, discovered in 1995 at the Tevatron, has proven to be a very interesting particle. Its properties allow to perform stringent tests of the Standard Model (SM) and to search for new physics through a deviation from SM predictions. We give here some expectations of what Tevatron run II will ultimately provide to our understanding of matter.

\section{INTRODUCTION}

After the success of the Fermilab Tevatron "Run I" (1992-1995), colliding protons and antiprotons at a center of mass energy $\sqrt{s}=1.8$ $\mathrm{TeV}$, the Tevatron Collider has undergone a massive upgrade. Also the CDF and DØ experiments completed a major upgrade. CDF has a new tracking system, with up to 8 silicon layers providing precise measurements of track parameters close to the beam line [1], and a new calorimeter for pseudorapidity $|\eta|>1[2]$. The trigger system and DAQ are completely new as well.

Tevatron Run II provided the first collisions at $\sqrt{s}=1.96 \mathrm{TeV}$ in march 2001. Almost one year was necessary to commission the new detector, and CDF started to collect physics-quality data in march 2002.

The analyses reported in the following are based on data sets taken until January 2003, corresponding to an integrated luminosity of 72 $\mathrm{pb}^{-1}$.

\section{W AND Z PRODUCTION}

$W$ and $Z$ bosons are produced at the Tevatron through $q \bar{q}$ annihilation. Only their leptonic decays are detected: $W \rightarrow e \nu, \mu \nu, \tau \nu$ and $Z \rightarrow e^{+} e^{-}, \mu^{+} \mu^{-}, \tau \tau$. The signature is given by high energy charged leptons and high missing transverse energy for $W$ candidates and two oppositely charged high energy leptons for $Z$ candidates.

\subsection{W Cross Section Measurements}

$W \rightarrow e \nu$ events are collected with a trigger selecting high $-E_{T}$ central electron candidates. After requiring one tight electron candidate with $E_{T}$ $>25 \mathrm{GeV}$ matched to a track of $P_{T}>10 \mathrm{GeV} / \mathrm{c}$ and missing transverse energy $E_{\mathrm{T}}>25 \mathrm{GeV}$, 38628 events remain in the data. The transverse mass spectrum $M_{T}$ of the candidate events and the estimated background is shown in figure 1 . The main background source is from QCD dijet events where a jet mimics the electron and large $\mathrm{Z}_{\mathrm{T}}$ is due to a second poorly measured jet. Additional backgrounds from $W \rightarrow \tau \nu$ or misidentified $Z \rightarrow$ ee decays are estimated from Monte Carlo simulations.

$W \rightarrow \mu \nu$ candidates are collected from high $-P_{T}$ muon triggers. After requiring an isolated muon with $P_{T}>20 \mathrm{GeV} / \mathrm{c}$ and $\mathrm{E}_{\mathrm{T}}>20 \mathrm{GeV}, 21599 \mathrm{~W}$ candidates are left (see figure 2). Backgrounds in this channel include misidentified boson decays, cosmic rays and QCD processes.

$W \rightarrow \tau \nu$ candidates can be selected by collecting events with a $\mathbb{Z}_{\mathrm{T}}>25 \mathrm{GeV}$ hardware trigger at level 1 and a subsequent software filter for $\tau$ identification at trigger level 3. Monojet candidates are kept if they have a single $E_{T}>25 \mathrm{GeV}$ jet containing one $P_{T}>4.5 \mathrm{GeV} / \mathrm{c}$ charged track in a $10^{\circ}$ cone and no other track within $30^{\circ}$, and 


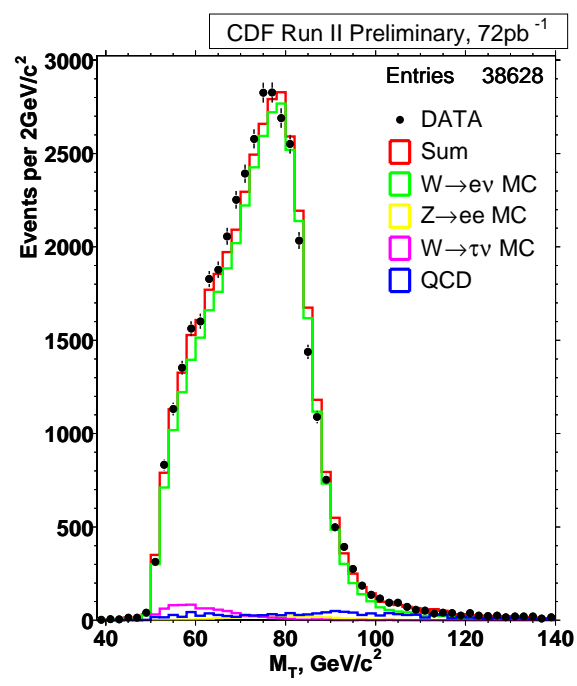

Figure 1. $W \rightarrow e \nu$ transverse mass $M_{T}$.

if $\mathrm{E}_{\mathrm{T}}>25 \mathrm{GeV}$. Electron events from $W \rightarrow e \nu$ decay are explicitly removed. 2345 events pass the selection. The background is mainly due to QCD processes. Additionally, using the excluded $W \rightarrow e \nu$ candidates in the same data sample, it is possible to extract the ratio of the electroweak coupling constants:

$g_{\tau} / g_{e}=0.99 \pm 0.04($ stat. $) \pm 0.07$ (syst. $)$.

A summary of the existing $g_{\tau} / g_{e}$ measurements is shown in figure 3 .

In Table 1 we summarize the measurement of the $\sigma \cdot B R(W \rightarrow \ell \nu)$ in the various channels (see also figure 4$)$. The results are in good agreement with NNLO calculations $(2.731 \pm 0.002 \mathrm{nb})[3]$.

The new forward electromagnetic calorimeter and tracking system, covering the region at $|\eta|>$ 1 made possible the identification of the $W \rightarrow$ $e \nu$ signal also at $|\eta|>1$ [4]. The corresponding transverse mass distribution is shown in Figure 5. The measurement of the cross section using these events is in progress.

\subsection{Z Cross Section Measurements}

The selection of $Z \rightarrow e^{+} e^{-}$candidates is done by requiring two central electrons with opposite charge, $E_{T}>25 \mathrm{GeV}$ and $P_{T}>10 \mathrm{GeV} / \mathrm{c}$. They must have invariant mass between 66 and 116

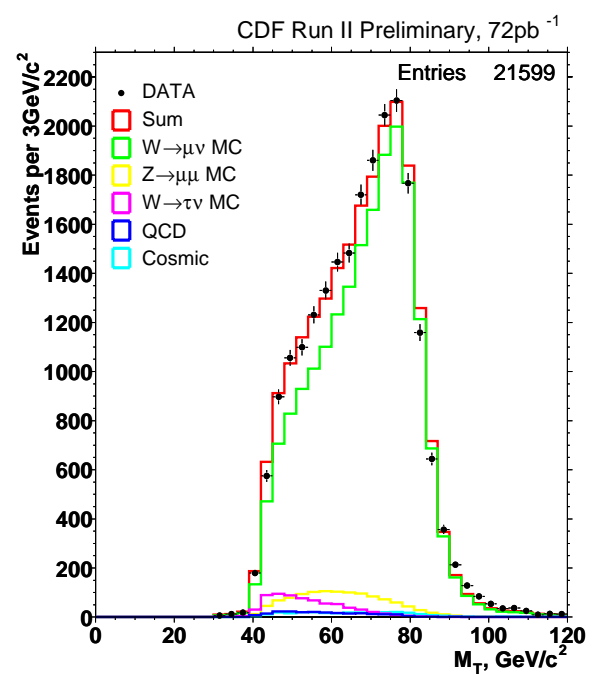

Figure 2. $W \rightarrow \mu \nu$ transverse mass $M_{T}$.

$\mathrm{GeV} / \mathrm{c}^{2} \cdot 1830 Z$ candidate events pass the selection. The invariant mass of these events is shown in figure 6 .

$Z \rightarrow \mu^{+} \mu^{-}$candidates are selected by requiring one isolated central muon with $P_{T}>20 \mathrm{GeV} / \mathrm{c}$ and a second isolated high- $P_{T}$ track passing minimum ionizing requirements. The same invariant mass window as in the electron channel is considered. $1631 Z$ candidate events are selected (see figure 7).

In Table 2 we summarize the measurement of the $\sigma \cdot B R\left(Z \rightarrow \ell^{+} \ell^{-}\right)$in the two channels (see also figure 8$)$. The results are consistent with NNLO calculations $(250.5 \pm 3.8 \mathrm{pb})[3]$.

The $Z \rightarrow \tau \tau$ signal is extracted in the channel where one $\tau$ decays into an electron (or a muon) and the other decays hadronically. In the electron-hadron channel the selection is made requiring a central electron with $E_{T}>10 \mathrm{GeV}$ and a hadronically decaying $\tau$-lepton with $E_{T}>$ $20 \mathrm{GeV}$, defined with the same criteria used in the $W \rightarrow \tau \nu$ analysis. We require in addition $M_{T}\left(e, \mathrm{Z}_{\mathrm{T}}\right) \leq 25 \mathrm{GeV} / \mathrm{c}^{2}$ and $P_{T}\left(e, \mathrm{Z}_{\mathrm{T}}\right) \geq$ $25 \mathrm{GeV} / \mathrm{c}$ to increase the purity of the signal. The track multiplicity for the selected hadronic tau candidates is shown in figure 9. Work is in progress to evaluate the cross section. 
Table 1

Summary of events selected in the various channels, expected background and corresponding $W$ production cross section.

\begin{tabular}{lccc}
\hline Channel & Events & Expec. Backgr. & $\sigma \times B R(W \rightarrow l \nu)(\mathrm{nb})$ \\
\hline$e \nu_{e}$ & 38625 & $6 \%$ & $2.64 \pm 0.02$ (stat.) \pm 0.09 (syst.) \pm 0.16 (lum.) \\
$\mu \nu_{\mu}$ & 21599 & $11 \%$ & $2.64 \pm 0.02$ (stat.) \pm 0.12 (syst.) \pm 0.16 (lum.) \\
$\tau \nu_{\tau}$ & 2346 & $26 \%$ & $2.62 \pm 0.07$ (stat.) \pm 0.21 (syst.) \pm 0.16 (lum.) \\
$e \nu_{e}, \mu \nu_{\mu}$ (combined) & & $2.640 \pm 0.012$ (stat.) \pm 0.093 (syst.) \pm 0.158 (lum.) \\
\hline
\end{tabular}

Table 2

Summary of events selected in the various channels, expected background and corresponding $Z$ production cross section.

\begin{tabular}{lccc}
\hline Channel & Events & Expec. Backgr. & $\sigma \times B R\left(Z \rightarrow l^{+} l^{-}\right)$(pb) \\
\hline$e^{+} e^{-}$ & 1830 & $0.6 \%$ & $267 \pm 6$ (stat.) \pm 15 (syst.) \pm 16 (lum.) \\
$\mu^{+} \mu^{-}$ & 1631 & $0.9 \%$ & $246 \pm 6$ (stat.) \pm 12 (syst.) \pm 15 (lum.) \\
$e^{+} e^{-}, \mu^{+} \mu^{-}$(combined) & & $251.5 \pm 4.3$ (stat.) \pm 10.6 (syst.) \pm 15.1 (lum.) \\
\hline
\end{tabular}

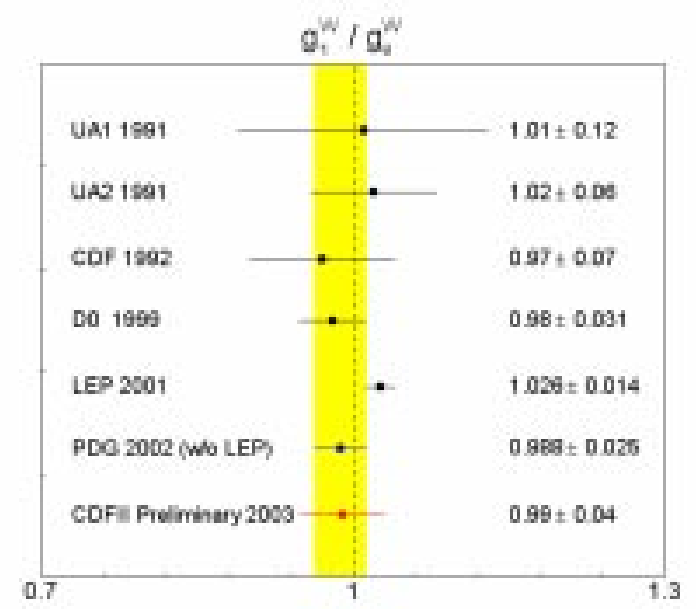

Figure 3. Summary of $g_{\tau}^{W} / g_{e}^{W}$ measurements.

\section{3. $\mathrm{W} / \mathrm{Z}$ Ratio and $\Gamma_{W}$ Extraction}

Using the cross section measurements presented in section 2.1 and 2.2 the ratio:

$R_{\ell}=\sigma_{W} B(W \rightarrow \ell \nu) / \sigma_{Z} B(Z \rightarrow \ell \ell)$

is computed to be:

$R_{e}=9.88 \pm 0.24($ stat. $) \pm 0.47$ (syst.)

and:

$R_{\mu}=10.69 \pm 0.27$ (stat.) \pm 0.33 (syst.).

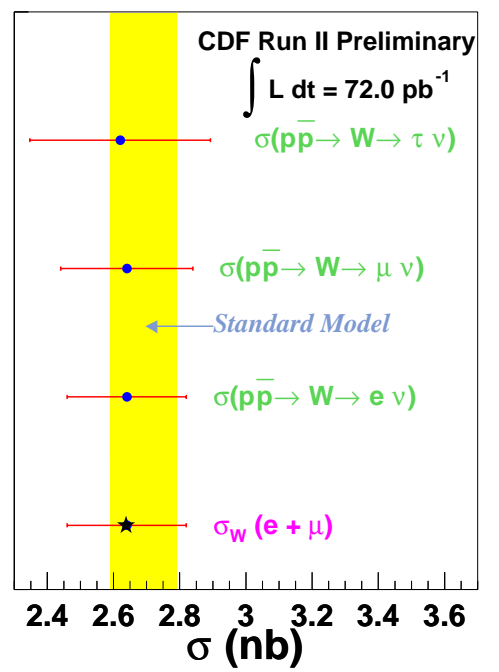

Figure 4. Combined $W$ cross section results.

An indirect determination of $\Gamma_{W}$ can be extracted from the $R$ measurement, using LEP measurements and theoretical predictions. The electron and muon channel results can be combined into:

$\Gamma_{W}=2146 \pm 78 \mathrm{MeV}$ 


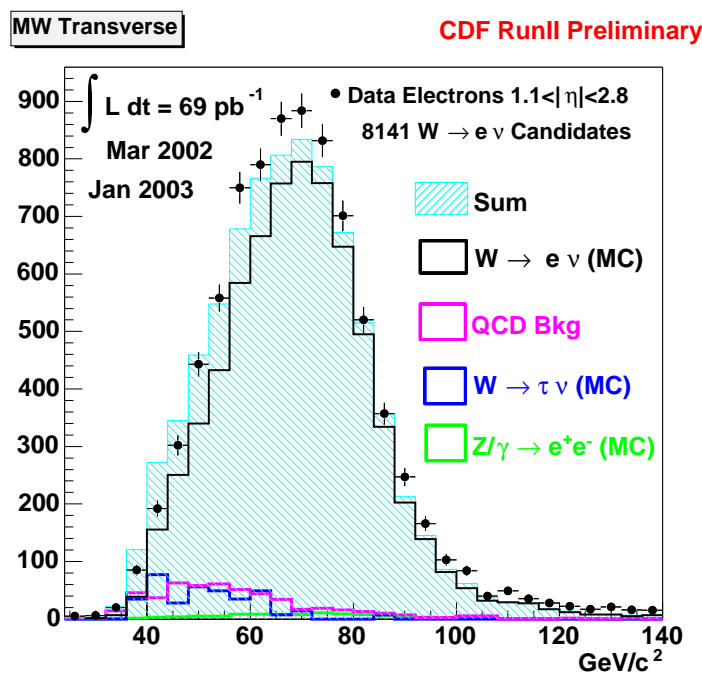

Figure 5. Transverse mass for $W$ candidate events where the electron is identified at $|\eta|>1$.

in relatively good agreement with the most recent average of $2118 \pm 42 \mathrm{MeV}$ [5] and the theoretical prediction of $2067 \pm 21 \mathrm{MeV}$.

\section{W MASS: PROSPECTS FOR RUN II}

Run II with $2 \mathrm{fb}^{-1}$ of data will provide each Tevatron experiment with over $3 \times 10^{6} \mathrm{~W}$ 's $(e$ and $\mu$ channel) therefore allowing a precise determination of many electroweak parameters. Since most of the systematic uncertainties on the $W$ mass $\left(M_{W}\right)$ measurement depends upon the size of the control samples, we will greatly benefit from the larger data set. The biggest contribution to the uncertainty comes from the momentum scale determination. This is obtained by fitting the $\ell^{+} \ell^{-}$ invariant mass spectrum. $W$ asymmetry is used to constrain $\mathrm{PDF}$ and the $Z P_{T}$ spectrum is the input to the $W P_{T}$ spectrum. With $2 \mathrm{fb}^{-1}$ we expect to reduce the overall uncertainty on $M_{W}$ to $40 \mathrm{MeV} / \mathrm{c}^{2}$, with $30 \mathrm{MeV} / \mathrm{c}^{2}$ as a possible target.

\section{TOP PAIRS PRODUCTION}

At the Tevatron energy $(\sqrt{s}=1.96 \mathrm{TeV})$ top quarks are produced primarily via the process $p \bar{p} \rightarrow t \bar{t}$. In the SM each top quark decays al-

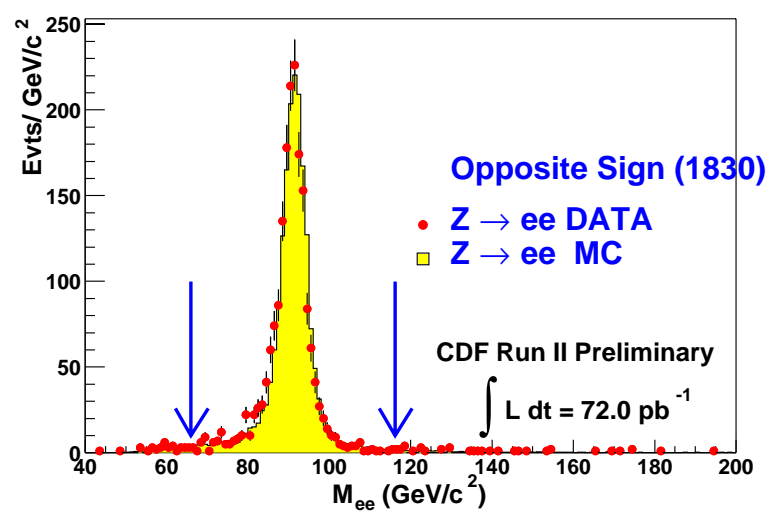

Figure 6. Invariant $Z \rightarrow e^{+} e^{-}$dilepton mass

most exclusively into a real $W$ and a $b$ quark $(t \rightarrow W b)$. Each $W$ subsequently decays into either a charged lepton and a neutrino or two quarks. The $t \bar{t} \rightarrow W^{+} b W^{-} \bar{b}$ events can thus be identified by means of different combinations of energetic leptons and jets. The branching ratio for both $W$ 's from a $t \bar{t}$ pair to decay leptonically is: $2 / 81$ for $e \mu, e \tau, \mu \tau$ and $1 / 81$ for $e e, \mu \mu, \tau \tau$ (dilepton channels). Decay modes of $t \bar{t}$ pairs in which one $W$ boson decays hadronically and the other leptonically (single lepton channel) have a branching ratio of $24 / 81$. When both $W$ 's decay hadronically (all hadronic channel) the branching ratio is $36 / 81$. CDF searched for the top quark using most of these signatures.

The first evidence for the presence of top events in the CDF data, reported in 1994 [6][7], was confirmed one year later by both the CDF and DØ Collaborations [8][9][10].

\section{TOP CROSS SECTION MEASURE- MENT}

By measuring the $t \bar{t}$ production cross section $\sigma_{t \bar{t}}$ in many channels and comparing it to perturbative QCD calculations, we can test the SM predictions in great detail.

\section{1. $\sigma_{t \bar{t}}$ in the dilepton channel}

Dilepton events are selected requiring two oppositely charged, isolated, high $P_{T}\left(P_{T}>20\right.$ 
Table 3

Preliminary run II results in the $t \bar{t}$ dilepton channel, for a dataset of $72 p b^{-1}$.

\begin{tabular}{lcccc}
\hline Source & $\mathrm{e}$ & $\mu \mu$ & $\mathrm{e} \mu$ & $\ell \ell$ \\
\hline Backgrounds & $0.103 \pm 0.056$ & $0.093 \pm 0.054$ & $0.100 \pm 0.037$ & $0.30 \pm 0.12$ \\
Expected $t \bar{t}$ & $0.47 \pm 0.05$ & $0.59 \pm 0.07$ & $1.44 \pm 0.16$ & $2.5 \pm 0.3$ \\
Data & 1 & 1 & 3 & 5 \\
\hline
\end{tabular}

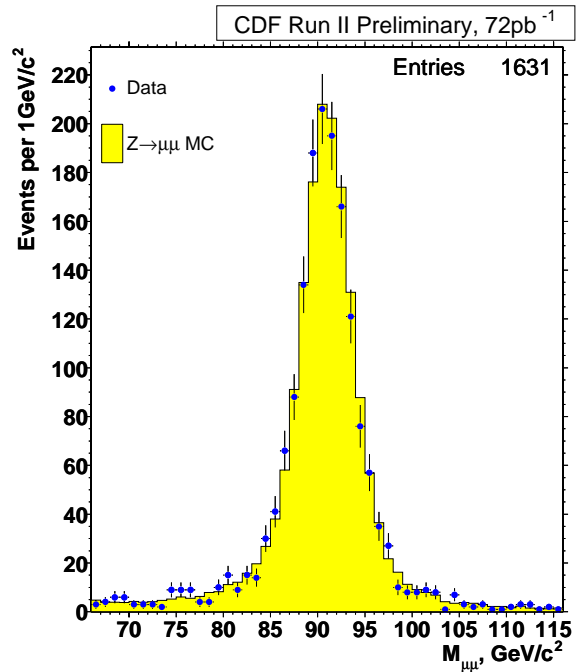

Figure 7. Invariant $Z \rightarrow \mu^{+} \mu^{-}$dilepton mass

$\mathrm{GeV} / \mathrm{c}$ ) leptons (e or $\mu$ ). Dielectron and dimuon events with invariant mass $M_{e e}$ or $M_{\mu \mu}$ in the interval $76-106 \mathrm{GeV} / \mathrm{c}^{2}$ are rejected, to reduce the $Z \rightarrow \ell^{+} \ell^{-} X$ background. Large missing transverse energy $\left(\mathbb{B}_{\mathrm{T}}>25 \mathrm{GeV}\right)$ is required. In addition, we require $\Delta \phi\left(\mathrm{E}_{\mathrm{T}}, \ell\right.$ or $\left.j\right)>20^{\circ}$ if $\mathrm{Z}_{\mathrm{T}}<50$ $\mathrm{GeV}$, to eliminate events with poorly measured $\mathrm{E}_{\mathrm{T}}$ due to mismeasured energies of lepton or jets, and to reduce $Z \rightarrow \tau \tau$ background. Two jets within $|\eta|<2$ and $E_{T}>10 \mathrm{GeV}$ are required also. Finally, to enhance the signal to background ratio, the scalar sum of the transverse energy of reconstructed objects in the event $H_{T}$ is required to be: $H_{T}>200 \mathrm{GeV}$. Figure 10 shows the dilepton candidates and the expectation from $t \bar{t}$ Monte Carlo for a top mass $M_{\text {top }}=175 \mathrm{GeV} / \mathrm{c}^{2}$. Table 3 summarizes the total background estimate, the expected signal and the number of observed events.

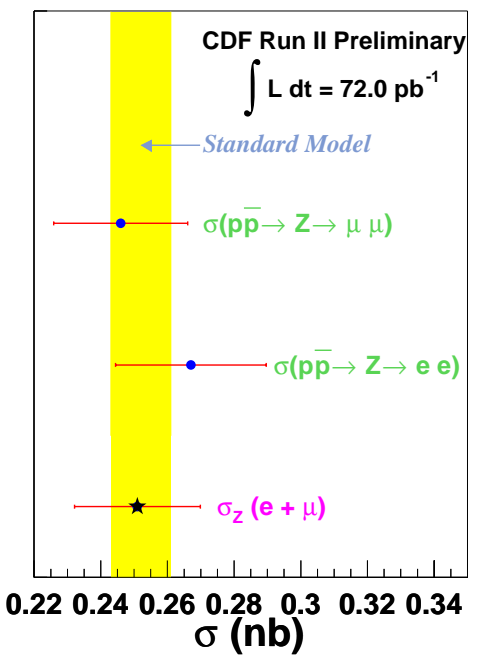

Figure 8. Combined $Z$ cross section results.

The cross section assuming $M_{t o p}=175 \mathrm{GeV} / \mathrm{c}^{2}$ is measured to be:

$\sigma_{t \bar{t}}=13.2 \pm 5.9$ (stat. $) \pm 1.5$ (syst. $) \pm 0.8($ lum. $) p b(5)$

which is higher but consistent with the NLO prediction: $6.70_{-0.88}^{+0.71} \mathrm{pb}[11]$.

\section{2. $\sigma_{t \bar{t}}$ in the lepton + jets channel}

The selection starts identifying $\mathrm{W}$ candidate events associated to jets within $|\eta|<2$ and $E_{T}>15 \mathrm{GeV}$. The 1 and 2 jet bins are used as control samples. Only $W+\geq 3$ jets are used to compute $\sigma_{t \bar{t}}$. To increase the signal to background ratio, the Silicon Vertex Detector SVX is used to identify b-quark displaced vertices. A jet is b-tagged if it contains a secondary vertex with at least two charged tracks and $L_{x y} / \sigma_{x y}>3$, where $L_{x y}$ is the distance in the transverse plane between the primary and secondary vertices and 


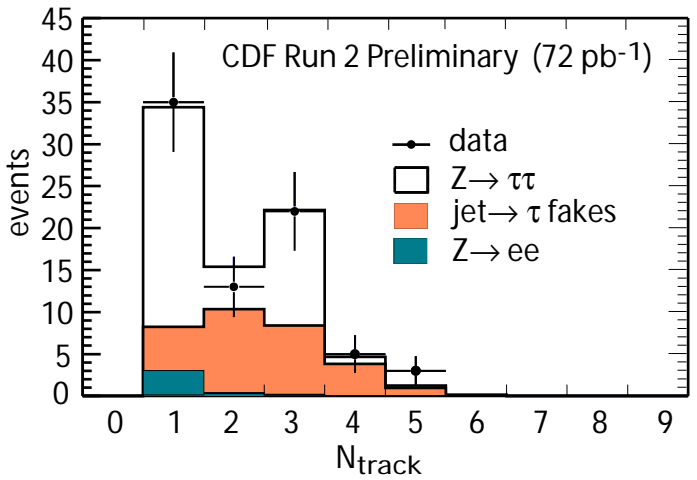

Figure 9. Multiplicity of tracks for selected hadronic tau candidates after baseline cuts. The data is compared to MC prediction assuming 250 pb cross section.

$\sigma_{x y}$ is the corresponding error.The efficiency for identifying at least one $\mathrm{b}$ quark in $t \bar{t}$ decays is about $45 \%$. The mistag rate is evaluated using the negative tag rate of $L_{x y}$. Table 4 summarizes the background estimate, with and without $t \bar{t}$ contribution, and the number of data candidates before and after requiring at least 1 jet with a b-tag.

From the 15 candidates with $\geq 3$ jets (see figure 11) the cross section is measured to be:

$\sigma_{t \bar{t}}=5.3 \pm 1.9$ (stat. $) \pm 0.8$ (syst. $) \pm 0.3($ lum. $) p b(6)$

which is consistent with the NLO prediction [11].

\section{TOP MASS MEASUREMENT}

The top quark mass is a fundamental parameter of the SM. Precise measurements of the top quark and $\mathrm{W}$ boson masses constraint the mass of the Higgs boson.

The most precise determination of the top mass is obtained from the single lepton plus jets channel. The sample is selected by requiring the presence of at least four jets in each event. In the first attempt of measuring the top mass in run II data CDF didn't ask for a b-tagged jet in the event. 33 events pass the selection, with an estimated background of 13 events. Each event in the sample is fitted to the hypothesis of $t \bar{t}$ production followed by decay in the lepton plus jets channel,

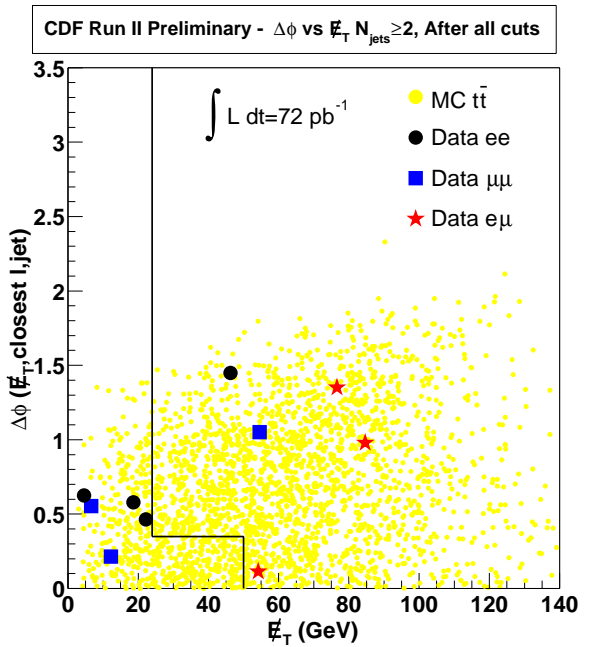

Figure 10. $t \bar{t}$ dilepton candidates found in 72 $\mathrm{pb}^{-1}$ in the plane $\Delta \phi\left(\mathbb{E}_{\mathrm{T}}, 1\right.$ or $\left.j\right)$ versus $\mathbb{Z}_{\mathrm{T}}$, in comparison with MC Herwig $t \bar{t}$ (yellow dots).

with a constraint fitting technique $\left(M_{l \nu}=M_{W}\right.$, $\left.M_{j j}=M_{W}, M_{t}=M_{\bar{t}}\right)$. Out of all the possible jet assignments we keep the solution which has the lowest fit $\chi^{2}$. The reconstructed top mass distribution from the data is then compared to the parametrized templates of top plus background. The top mass is extracted by fitting the observed and expected distributions using a maximum likelihood method (see figure 12). The measured top mass is:

$M_{\text {top }}=171_{-12.5}^{+14.4}$ (stat. $) \pm 9.9$ (syst. $) \mathrm{GeV} / \mathrm{c}^{2}$.

The main systematic uncertainty comes from the jet energy measurement, which is currently 9.3 $\mathrm{GeV} / \mathrm{c}^{2}$, compared to $4.4 \mathrm{GeV} / \mathrm{c}^{2}$ in run I. CDF aims to reduce this error to $\approx 2 \mathrm{GeV} / \mathrm{c}^{2}$.

\section{TOP PHYSICS: PROSPECTS FOR RUN II}

After the top discovery the Tevatron experiments moved to detailed studies of its properties. Both experiments investigated the top candidate events kinematic properties and the decay vertex. CDF did an indirect measurement of the $V_{t b}$ 
Table 4

Preliminary run II results in the $t \bar{t}$ lepton + jets analysis with displaced vertex b-tagging, for a dataset of $57.5 p b^{-1}$.

\begin{tabular}{lcccc}
\hline Source & $\mathrm{W}+1$ jet & $\mathrm{W}+2$ jets & $\mathrm{W}+3$ jets & $\mathrm{W}+\geq 4$ jets \\
\hline SM Background & $33.8 \pm 5.0$ & $16.4 \pm 2.4$ & $2.88 \pm 0.05$ & $0.87 \pm 0.2$ \\
SM Background $+t \bar{t}$ & $34.0 \pm 5.0$ & $18.65 \pm 2.4$ & $7.35 \pm 1.4$ & $7.62 \pm 2.0$ \\
Data before b-tagging & 4913 & 768 & 99 & 26 \\
Data ( $\geq 1 \mathrm{~b}-$-tag) & 31 & 26 & 7 & 8 \\
\hline
\end{tabular}

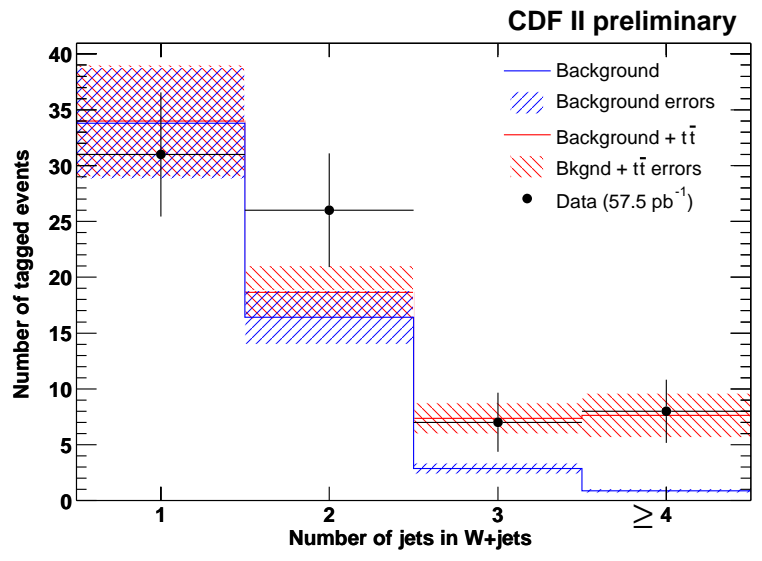

Figure 11. Number of events in the $\mathrm{W}+$ jets sample with at least one $\mathrm{b}-$ tag. $\mathrm{W}+\geq 3$ jets are used to extract $\sigma_{t \bar{t}}$.

vertex [12], studied the $W$ boson helicity in top decays [13], the top $P_{T}$ distribution [14] and the $t \bar{t}$ system invariant mass [15]. DØ studied the top-antitop spin correlations and other kinematic features [16]. Most of these studies were statistically limited. However, they can be viewed as a preview of possibilities for the Tevatron in run II.

The single top production mechanism involves electroweak production of a single top via the $W t b$ vertex, with about half production cross section than the pair production and much larger background. The single top mode was not observed in run I (both experiments set upper limits on single top production cross section [17] [18]) and it is one of the goals of run II. In Table 5 we summarize the projection of top quark measurements for run II, assuming that $2 \mathrm{fb}^{-1}$ of data will be

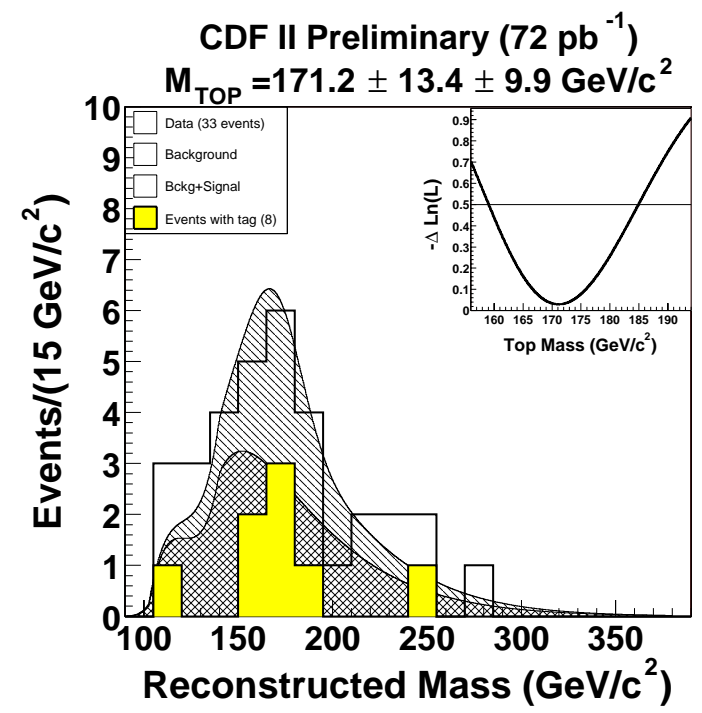

Figure 12. Reconstructed top mass distribution of pre-tagged lepton +4 jet events. The yellow histogram represents events with a b-tag, but the information is not used in the top mass reconstruction.

collected.

\section{CONCLUSIONS}

The $W, Z$ and $t \bar{t}$ cross sections have been measured by CDF using the first run II physics quality data. The results shown here, based on 72 $\mathrm{pb}^{-1}$, are in agreement with theoretical predictions at $1.96 \mathrm{TeV}$.

At the time of this writing the CDF experiment has collected about $170 \mathrm{pb}^{-1}$ of which about 120 $\mathrm{pb}^{-1}$ have been already analized. Preliminary re- 
Table 5

Summary of achieved precision for top quark measurements and projections for run II.

\begin{tabular}{lccc}
\hline Top quark Property & Run I measurement & \multicolumn{2}{c}{ Precision } \\
\hline & & Run I & Run II \\
\hline Mass $(\mathrm{CDF}+\mathrm{D} \varnothing)$ & $174.3 \pm 3.2 \pm 4.0$ & $2.9 \%$ & $1.2 \%$ \\
\hline$\sigma_{t \bar{t}}(\mathrm{CDF} 175 \mathrm{GeV})$ & $6.5_{-1.4}^{+1.7}$ & $25 \%$ & $10 \%$ \\
$\sigma_{t \bar{t}}(\mathrm{D} \varnothing \quad 172 \mathrm{GeV})$ & $5.69 \pm 1.21 \pm 1.04$ & $28 \%$ & $10 \%$ \\
\hline $\mathrm{W}$ helicity, $\mathrm{F}_{0}$ & $0.91 \pm 0.37 \pm 0.13$ & 0.4 & 0.09 \\
$\mathrm{~W}$ helicity, $\mathrm{F}_{+}$ & $0.11 \pm 0.15 \pm 0.06$ & 0.15 & 0.03 \\
\hline$\left|V_{t b}\right|$ & $0.97_{-0.12}^{+0.16}(3-$ gen $)$ & $15 \%$ & $3 \%$ \\
\hline$\sigma($ single top $)$ & $\left|V_{t b}\right|>0.6190 \% \mathrm{CL}$ & & $20 \%$ \\
$\Gamma(t \rightarrow W b)$ & $<14$ pb & - & $25 \%$ \\
$\left|V_{t b}\right|($ direct from $\sigma)$ & - & - & $12 \%$ \\
\hline $\mathrm{BR}(t \rightarrow \gamma q) 95 \% \mathrm{CL}$ & - & 0.03 & $2 \times 10^{-3}$ \\
$\mathrm{BR}(t \rightarrow Z q) 95 \% \mathrm{CL}$ & 0.03 & 0.30 & 0.02 \\
\hline
\end{tabular}

sults are encouraging and indicate that the CDF upgraded detector will be able to fully exploit the available physics opportunities.

Till now the Standard Model had a remarkable success in describing the elementary particle interactions. The Higgs sector however remains unexplored. The uncertainties expected in run II (assuming $2 \mathrm{fb}^{-1}$ of data) on the measurement of $M_{W}$ and $M_{t o p}$ should lead to a constraint of about $40 \%$ on the Higgs mass.

\section{REFERENCES}

1. A. Sill et al., Nucl.Instrum.Meth. A447, 1 (2000); A. Affolder et al., Nucl.Instrum.Meth. A485, 6 (2002).

2. R. Blair et al., FERMILAB-PUB-96/390-E (1996).

3. R. Hamberg, W.L. Van Neerven and T. Matsuura, Nucl. Phys.B359,343 (1991);

R.V. Harlander and W.B. Kilgore, Phys. Rev. Lett. 88, 211801 (2002).

4. A. Sidoti, to appear in EPS03 proceedings.

5. K. Hagiwara et al., Phys.Rev. D66, 010001 (2002).

6. F. Abe et al.,Phys.Rev.Lett.73,225(1994); F. Abe et al, Phys.Rev.D50,2966(1994).

7. F. Abe et al, Phys.Rev.D51,4623(1995).

8. F. Abe et al.,Phys.Rev.Lett.74,2626(1995).
9. F. Abe et al.,Phys.Rev.D52,2605(1995).

10. S. Abachi et al.,Phys.Rev.Lett.74,2632(1995).

11. M. Cacciari et al.,CERN-TH-2003-054 (hep$\mathrm{ph} / 0303085$ ).

12. T. Affolder et al., Phys. Rev. Lett. 86, 3233 (2001).

13. T. Affolder et al., Phys. Rev. Lett. 84, 216 (2000).

14. T. Affolder et al., Phys. Rev. Lett. 87, 102001 (2001).

15. T. Affolder et al., Phys. Rev. Lett. 85, 2062 (2000).

16. B. Abbott et al., Phys. Rev. Lett. 85, 256 (2000).

17. D. Acosta et al.,Phys. Rev. D64, 091102 (2002).

18. V.M. Abazov et al.,Phys. Lett. B517, 282 (2001). 